\title{
FILIACIÓN, RELACIONES INTERPOBLACIONALES Y ENLACES CULTURALES EN LAS TIERRAS BAJAS MAYAS DURANTE EL PERIODO CLÁSICO
}

\author{
VerA TIESLER \\ AndREa CuCINA \\ Facultad de Ciencias Antropológicas, \\ Universidad Autónoma de Yucatán
}

\begin{abstract}
Resumen: La presente investigación se dedica al análisis morfológico de piezas dentales y de craneos deformados de poblaciones mayas que habitaron en las Tierras Bajas durante el periodo Clásico; el objetivo es reconstruir las dinámicas poblacionales, así como las afinidades biológicas y culturales. Los restos proceden de una serie de sitios ubicados en el Petén mexicano y guatemalteco; fueron estudiados tomando en cuenta un contexto regional y comparándolos con los datos de varios sitios coetáneos de Chiapas, Yucatán y Quintana Roo. Los datos dentales fueron analizados estadísticamente por medio del análisis de agrupación y de máxima semejanza mientras que los datos craneanos se evaluaron según las proporciones de presencia y tipos de deformación cultural. Los resultados evidencian una continuidad poblacional y cultural en un eje norte-sur entre los grupos clásicos del Petén norte y central, y de manera notablemente menor hacia los sitios coetáneos al oeste del Usumacinta.
\end{abstract}

Palabras Clave: Tierras Bajas Mayas, biodistancias, modificación cefálica, movimientos poblacionales, Calakmul.

Aвstract: The present study evaluates the dental and artificial cranial morphology of Classic period Lowland Mayas to reconstruct their populational dynamics, biological and cultural affinities. The remains come from several sites located in the Mexican and Guatemaltecan Peten area. The data were elaborated within their regional contexts and compared with information derived from coeval settlements from Chiapas, Yucatan, and Quintana Roo. The dental data were analyzed statistically using cluster and Maximum Likelihood analyses, while the cranial information was processed comparing proportions of presence and type of vault modification. The results evidence populational and cultural continuity in Peten's north-south axis during the Classic period and to a much minor degree also towards occupations west of the Usumacinta river.

KeYwords: Lowland Mayas, biodistance, cranial modification, population movements, Calakmul.

RECEPCIÓN: 25 de junio del 2010.

ACEPTACIÓN: 29 de junio del 2011. 



\title{
FILIACIÓN, RELACIONES \\ INTERPOBLACIONALES Y ENLACES \\ CULTURALES EN LAS TIERRAS BAJAS MAYAS DURANTE EL PERIODO CLÁSICO
}

\author{
Vera TIESLER \\ AndREa CUCINA \\ Facultad de Ciencias Antropológicas, \\ Universidad Autónoma de Yucatán
}

\section{Introducción}

El conocimiento integral de las culturas pretéritas requiere de un acercamiento multidisciplinario que sea capaz de dilucidar sus expresiones desde varios ángulos analíticos y campos de conocimientos. Este fue el punto de partida del presente trabajo que pretende rastrear la trayectoria de la gran ciudad de Calakmul y su Reino de Kaan, del que una vez fue capital, desde una perspectiva que no se ancla en sus vestigios materiales o su herencia gráfica, sino en la población calakmulense misma y su convivencia con otros grupos de las Tierras Bajas durante el periodo Clásico. ${ }^{1}$

Hasta la fecha, los estudios que abordan las poblaciones mayas prehispánicas han sido fundamentados mayormente en el registro escrito y en la cultura arqueológica, ya sea el patrón de asentamiento o la paleodemografía cuantitativa, la arqueología doméstica o las fuentes escritas sobre linajes, lenguas, territorios y relaciones políticas (Culbert y Rice, 1990; Lacadena y Wichman, 2002; Sharer y Traxler, 2006; Martin y Grube, 2008; Martínez Muriel, 2008). Un acercamiento más directo, aunque proble-

\footnotetext{
"Deseamos agradecer a todos los colegas y proyectos que facilitaron la colección de datos. Estamos agradecidos especialmente con los siguientes proyectos e instituciones por su apoyo, asesoría y colaboración: al Proyecto Arqueológico Calakmul (Ramón Carrasco, Instituto Nacional de Antropología e Historia), al Proyecto Arqueológico Calakmul (William Folan, Universidad Autónoma de Campeche), al Proyecto Arqueológico Sur de Quintana Roo (Enrique Nalda, INAH), al Proyecto Arqueológico Xcambó (Thelma Sierra, Centro INAH Yucatán), al Proyecto Arqueológico Noh Bec, Cono Sur de Yucatán (Agustín Peña, Centro INAH Yucatán), a la Dirección de Antropología Física (José Antonio Pompa, INAH), a la Coordinación de Museos del centro INAH Campeche (Marco Antonio Carvajal), al Proyecto Atlas Arqueológico de Guatemala (Juan Pedro Laporte, Instituto de Antropología e Historia). Asimismo deseamos expresar nuestras gracias a las organizadoras del Simposio en el Congreso Internacional de Americanistas (2009) que dio pie a este trabajo, a Verónica Vázquez y Ana García.

${ }^{1}$ Una versión preliminar de este trabajo fue presentado como ponencia en el 53을 Congreso Internacional de Americanistas en el 2008 (Simposio: "Nuevas perspectivas en torno a la organización sociopolítica de las Tierras Bajas del Sur en el Clásico Tardío: El Reino de Kaan”).
} 
mático, es el estudio de la estructura poblacional interna de cada sitio, denominada "paleodemografía cualitativa". Esta se funda en la interpretación de la distribución de sexos y grupos de edades en colecciones de esqueletos (Civera y Márquez, 1996; véase también Hoppa y Vaupel, 2002). Su evaluación permite inferir la mortalidad relativa en relación con el sexo y los grupos de edad, así como la fertilidad de un grupo (Sattenspiel y Harpending, 1983). Desafortunadamente, el éxito de este acercamiento (al igual que el de otros estudios bioarqueológicos) depende directamente de la preservación de los restos humanos y el tamaño de las series estudiadas, dos factores que se traducen en claras limitantes en las Tierras Bajas Mayas, donde predomina el deterioro de todo vestigio arqueológico de origen orgánico. Son problemáticas también las posibilidades actuales de determinar el sexo y, sobre todo, de asignar estimaciones crono-vitales correctas en cada individuo (Hoppa y Vaupel, 2002).

En esta contribución deseamos, por tanto, abordar el tema de la población de Calakmul a partir de la tipología morfológica de sus pobladores; del mismo modo abarcamos también las relaciones entre poblaciones y los enlaces culturales de Calakmul con sus vecinos. Para su comparación en el marco regional, privilegiamos un acercamiento combinado (i.e. a través de la morfología dental y las modificaciones artificiales de la cabeza) en series de esqueletos mayas según su contexto espacial, cronológico y cultural. Este trabajo se construye directamente sobre los resultados obtenidos en una serie de otros estudios propios desarrollados en la región (Cucina, Tiesler y Wrobel, 2005; Cucina y Tiesler, 2008; Tiesler, 1998, 1999; Tiesler, 2011). Estos examinan por separado la morfología dental y las modificaciones artificiales de la cabeza en la región maya, dos atributos bioculturales que en este estudio deseamos estudiar conjuntamente.

Así planteado, evaluamos primero la estructura morfológica dental de la población calakmulense y aquella de los asentamientos mayas coetáneos, eso a partir de la distribución de sus características no métricas en dientes permanentes. Siendo producto de las dinámicas microevolutivas poblacionales, su estudio promete brindar una nueva visión de la variabilidad interna de los calakmulenses y de otros grupos humanos de la región (Cucina y Tiesler, 2008; Cucina, Tiesler y Webber, 2005). Posteriormente rastreamos algunas tendencias en sus relaciones con otras poblaciones y las comparamos con la distribución regional de las formas artificiales de la cabeza. Las preferencias en esta práctica corporal emblemática, según proponemos, pudieran expresar pertenencias clánicas y posiblemente étnicas que se dieron a lo largo del corredor geográfico petenero y sus vecinos (Tiesler, 2011).

\section{Materiales y métodos}

\section{La muestra}

Para los objetivos que competen a este estudio, hemos analizado una serie de colecciones esqueléticas mayas del periodo Clásico (véase la figura 1 para la 


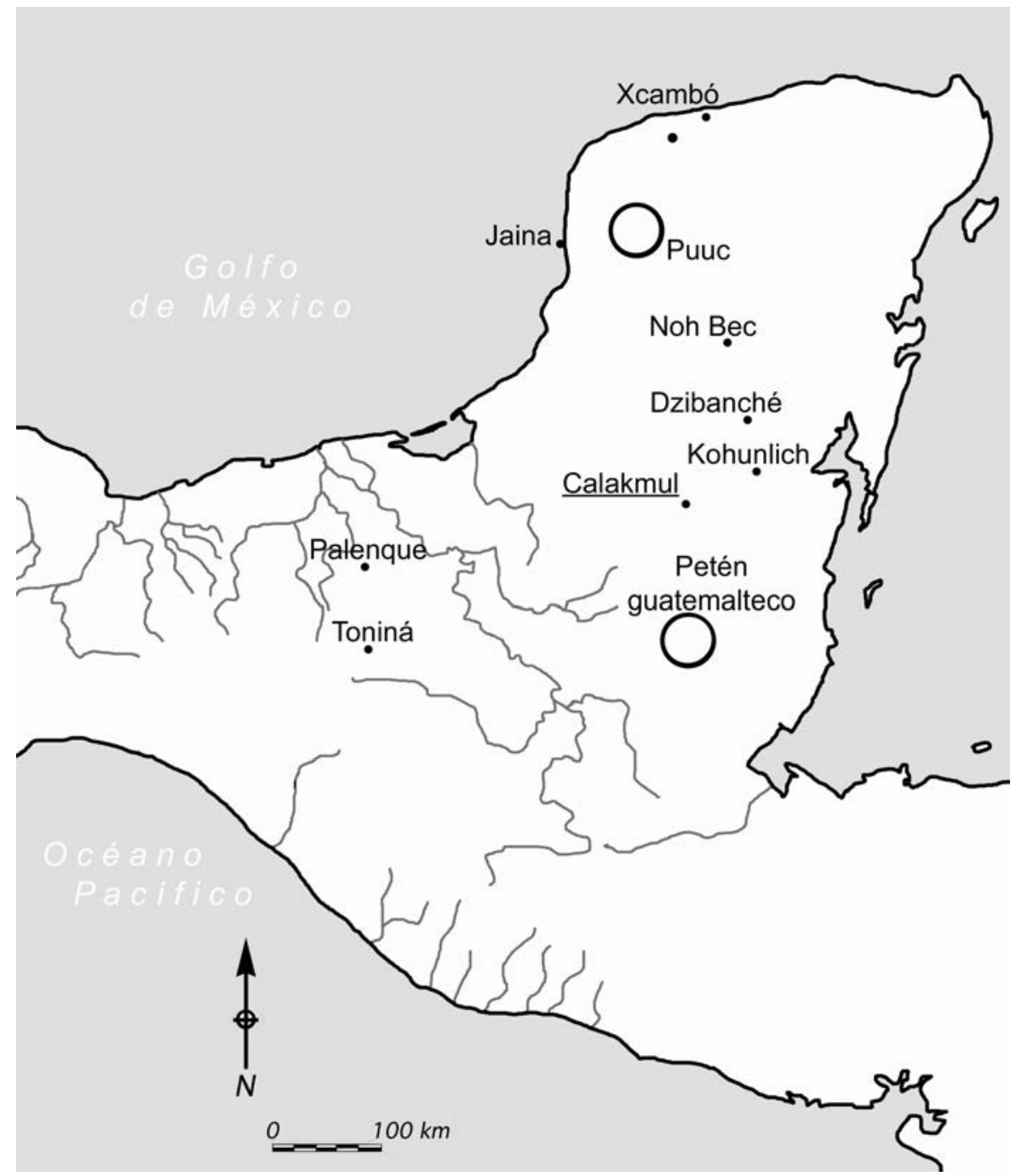

FiguRA 1. Mapa del área maya con sitios y regiones estudiadas para el presente artículo

identificación y ubicación geográfica de las colecciones estudiadas). La muestra total cubre estudios de sitios arqueológicos del sureste de México y Chiapas, Belice y Guatemala; asimismo, la franja noroeste de Honduras. Los datos específicos que reunimos y combinamos en este estudio han sido acumulados a lo largo de muchos años de estudio: ahora integran información de un total de 1350 osamentas, todas fechadas para el Clásico. De este total, unos 615 esqueletos cuentan con datos dentales y unos 722 individuos óseos podían ser evaluados según la práctica de modificación cefálica que presentaran. 
Arqueológicamente hablando, el total incluye 73 individuos recabados por los dos proyectos que han explorado la ciudad de Calakmul (Universidad Autónoma de Campeche e Instituto Nacional de Antropología e Historia), además de las series esqueléticas de los sitios cercanos al este: Dzibanché (46 individuos) y Kohunlich (55 individuos) en el actual estado de Quintana Roo (Proyecto Sur de Quintana Roo, INAH). ${ }^{2}$ Más al sur, reunimos una serie de osamentas que denominamos Petén Guatemalteco (Proyecto Atlas Arqueológico de Guatemala), dividido en Clásico Temprano (39 individuos) y Tardío (102 individuos). Al suroeste de Calakmul registramos las series de Palenque (representado por 39 individuos, INAH) y de Toniná (33 individuos, Centro de Estudios Mexicanos y Centroamericanos). Más al norte cuantificamos los patrones observados en el sitio de Noh Bec (41 individuos, INAH), un centro de transición recién excavado en el centro de la península yucateca, además de un consorcio de tres sitios (Chac, Siho y Oxkintok/ INAH, National Geographic y Universidad Autónoma de Yucatán) que denominamos Puuc, y que suman un total de 24 individuos. Incluimos también dos sitios clásicos costeros que arrojaron poblaciones funerarias abundantes, que son Jaina (96 individuos, INAH) y Xcambó (67 individuos, INAH). Para fines comparativos en las elaboraciones estadísticas de medición, recurrimos a una muestra externa de control, independiente y alejada de las muestras a examinar para este estudio (outlier). Esta pertenece al periodo neolítico europeo (Neo) (Cucina, datos personales).

\section{La deformación cefálica intencional}

Contrario a la connotación de "moda" que se le ha dado a la modificación cultural de la cabeza infantil, es una práctica que trasciende las generaciones al ser practicada por mujeres de segunda o tercera generación en recién nacidos que después portaban el resultado visual el resto de su vida (Torres-Rouff, 2002; Blom, 2005). En el ámbito maya prehispánico, la deformación fue parte integral del repertorio cultural por al menos tres milenios. Evaluada a partir de los vestigios craneanos, la tradición es apta para identificar aspectos de identidad y cambio cultural de mediano y largo plazo. En estudios anteriores (Tiesler, 1998, 1999; Tiesler, 2011) se ha concluido que la costumbre, que dejó sus huellas en más del 80 por ciento de los cráneos, preparaba a los infantes para su posterior integración social y religiosa, sancionada mediante una serie de ritos de iniciación infantil (véase también Bonavides, 1992: 404-406). En particular durante el periodo Clásico, con su impresionante diversidad de formas artificiales de la cabeza (figura 2), los resultados visibles deben haber tenido un valor emblemáti-

\footnotetext{
${ }^{2}$ Hemos dividido la serie de Kohunlich en dos grupos siguiendo el consejo de Enrique Nalda: el primero derivado de 27 Escalones (Kohunlich 27, representado por 33 individuos) y el otro denominado "grupo restante" (Kohunlich, representado por 22 individuos). La división igualmente nos sirve para tener un indicio de la diversidad interna del grupo y como control.
} 

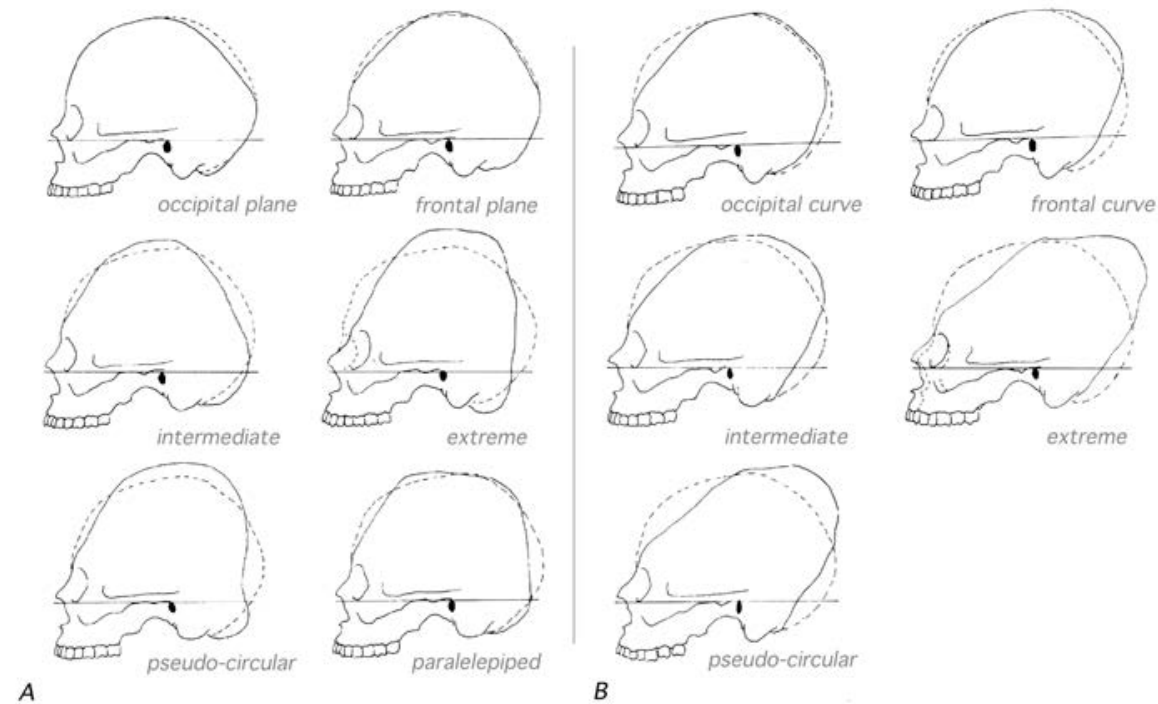

Figura 2. Diversidad de formas cefálicas, acorde con la clasificación empleada:

(A) variantes de modificaciones tabulares oblicuas;

(B) variantes de modificaciones tabulares erectas

co, expresando identidades en conformidad con esquemas ideológicos vigentes (Romano, 1987; Sotelo y Valverde, 1992; Tiesler, 1998; Houston, Stuart y Taube 2006: 45). Tal parece que las habilidades del artificio se pasaban de generación en generación en cada familia, generando la asombrosa variedad de formas cefálicas que se observa en la colectividad (Tiesler, 1999, 2005).

Para los fines de este trabajo nos hemos enfocado en las distribuciones formales en cada serie bajo estudio. Para la clasificación se empleó la tipología propuesta por Dembo e Imbelloni (1938), Romano (1965) y modificada por Tiesler (1998, 1999 y 2011), que distingue dos tipos principales: una, del tipo tabular erecta, la cual era lograda mediante la compresión de la cabeza en cunas; y otra, tabular oblicua, alcanzada gracias a la colocación de tablones sobre frente y occipucio de la cabeza infantil (figuras 2 y 3 ). Aparte se registraron diferentes grados y variantes de cada técnica.

\section{Estudio dental}

Los rasgos morfológicos dentales corresponden a todas las manifestaciones con un componente genético y que varían entre poblaciones. Aquellos quedan expresados en forma de surcos, crestas, tubérculos, presencia o tamaño de cúspides, 

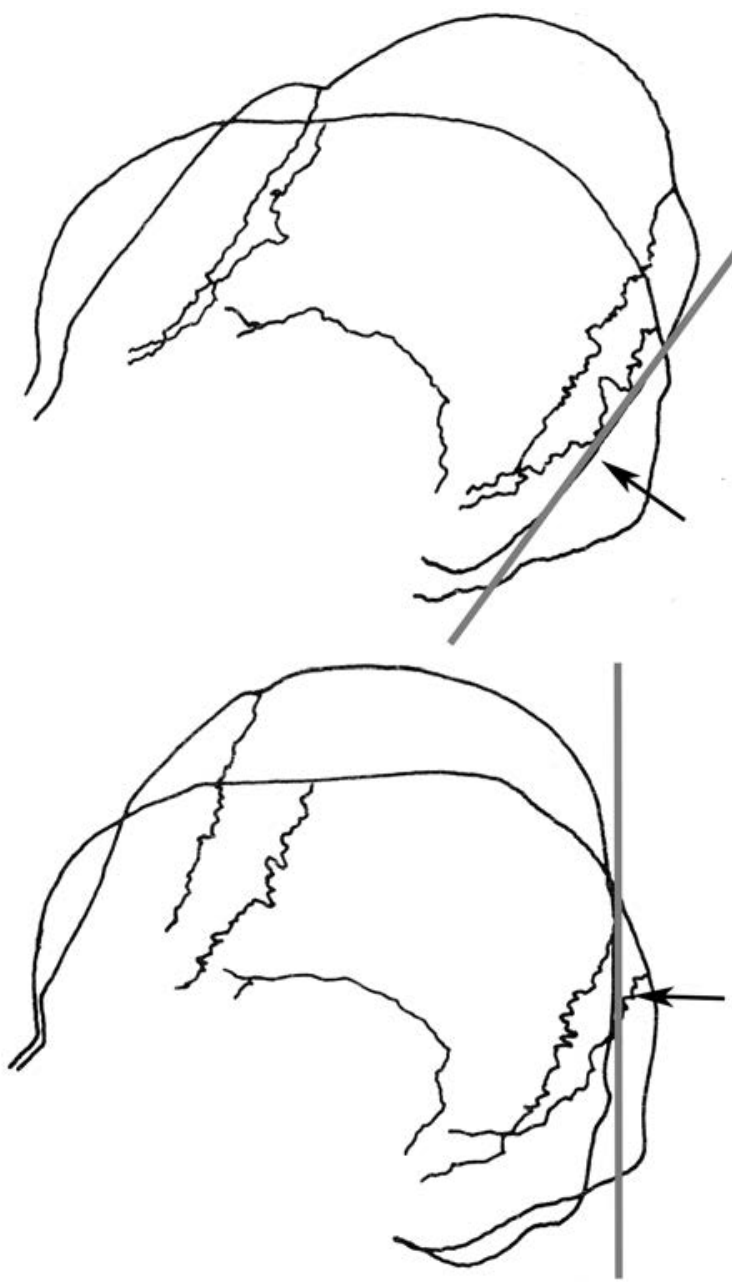

FiguRa 3. Vectores en los planos de compresión posterior: cráneo tabular oblicuo (arriba) y cráneo tabular erecto (abajo) (trazado de Dembo e Imbelloni, 1938: 50)

número de cúspides, de raíces, etc. Estudios recientes han mostrado que los rasgos morfológicos dentales brindan importante información sobre los procesos microevolutivos (Scott y Turner, 1997). A principios de los años noventa, Christy Turner y sus colegas (1991), actualizando y ampliando una serie de estándares anteriores (Dahlberg, 1956) desarrollaron la clasificación para registrar los rasgos morfológicos dentales comúnmente utilizada en estudios de antropología dental y conocida con la sigla de ASUDAS (Arizona State University Dental Anthropology System). Este método tiene un elevado potencial discriminatorio en los análisis de afinidades biológicas micro y macroregionales (Scott y Turner, 1997). Su 
potencialidad discriminatoria deriva del elevado número de rasgos que abarca (Scott y Turner, 1997), del reducido ritmo evolutivo -que permite estudios diacrónicos de amplia escala (Turner, 1969)—, de la baja influencia, en comparación con otros tejidos, que los factores ambientales ejercen en la manifestación de los rasgos (con la sola excepción del desgaste; Larsen y Kelley, 1991), y sobre todo, de su elevado componente hereditario (Nichol, 1989).

Además, los dientes constituyen la parte más dura de todo el esqueleto (Hillson, 1996), lo que les proporciona una resistencia considerable a las bien conocidas condiciones extremosas que sufren los restos arqueológicos humanos en el área maya; por tanto, las piezas dentales son los únicos elementos disponibles que pueden proporcionar información útil para análisis esqueléticos.

Por otra parte importa subrayar que, debido a la variabilidad interna de las poblaciones y en un contexto microregional como el abarcado en este estudio, no es posible hacer inferencias individuales. Los rasgos dentales evaluados para fines de medición de afinidad y estructura biológica sólo trazan tendencias generales. Otra limitante radica en el error entre observadores, problema que aquí se trató de eliminar al no incluir en este estudio datos morfológicos de otros autores.

Para este estudio, los rasgos fueron registrados en todos los dientes permanentes según la metodología AsUDAs. En los casos en los que el mismo diente se encontraba presente en ambos lados (izquierdo y derecho), sólo fue contabilizada la pieza con mayor expresión. Los datos crudos comprenden un total de 79 atributos. Cabe agregar que de los 79 rasgos fueron utilizados solo 33 para las comparaciones estadísticas entre series (tabla 1), tras eliminar todos los atributos claramente correlacionados (por ejemplo, la pala del incisivo central y lateral) y otros que mostraban una reducida variabilidad interpoblacional. Los rangos de expresión fueron transformados en frecuencias dicotómicas de presencia o ausencia.

Seguidamente se calculó la diversidad fenotípica interna de cada serie por medio del índice de heterocigosidad, un valor comúnmente usado en las ciencias biológicas para expresar la variabilidad de las características morfológicas entre individuos. Se calcula como el promedio de los valores de variabilidad de cada rasgo, según la fórmula que aquí se presenta:

Índice de heterocigosidad: $\mathrm{IH}=\sum\left\{\mathrm{n}_{\mathrm{i}}\left[1-\left(\mathrm{x}_{\mathrm{i}}^{2}+\mathrm{y}_{\mathrm{i}}{ }^{2}\right)\right] /\left(\mathrm{n}_{\mathrm{i}}-1\right)\right\} / \mathrm{N}^{3}$

Índices elevados (que se acercan a 0.5) indican poblaciones abiertas, variables y exógamas, típicas de grandes agrupaciones poblacionales tales como los resi-

\footnotetext{
3 - $i$ es el rasgo individual;

- $X$ es la frecuencia de presencia del rasgo $\mathrm{i}$;

- $Y$ es la frecuencia de ausencia del rasgo i (1-x);

- $n_{i}$ es el total de dientes disponibles para el rasgo i;

- $N$ es el total de 79 rasgos.
} 


\begin{tabular}{|c|c|c|c|c|c|c|c|c|c|c|c|c|c|c|c|c|c|}
\hline $\begin{array}{l}\stackrel{\circ}{0} \\
\text { 芠 }\end{array}$ & $\hat{\infty}$ & $\frac{m}{6}$ & 疋 & 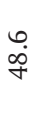 & 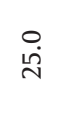 & $\begin{array}{l}\infty \\
\stackrel{\omega}{\omega}\end{array}$ & $\overline{\dot{D}}$ & $\stackrel{\tilde{m}}{\ddot{\nu}}$ & $\stackrel{亏}{\bar{F}}$ & g̊̀ & $\stackrel{\Xi}{\bar{\sim}}$ & กֶ. & $\stackrel{\infty}{m}$ & $\stackrel{\circ}{\stackrel{+}{N}}$ & $\begin{array}{l}0 \\
\dot{0} \\
\dot{\phi}\end{array}$ & & $\hat{m}$ \\
\hline 芚 & ஜ્ & $\stackrel{\circ}{\circ}$ & 节 & ঙ̆ & 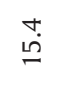 & $\stackrel{\mathscr{m}}{\dot{ \pm}}$ & 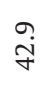 & $\stackrel{\Re}{ \pm}$ & $\stackrel{\text { n? }}{\stackrel{n}{m}}$ & $\frac{1 ?}{6}$ & $\begin{array}{l}\stackrel{+}{0} \\
\text { மे }\end{array}$ & $\stackrel{\stackrel{n}{\sim}}{\tilde{\nu}}$ & $\stackrel{m}{m}$ & $\stackrel{\circ}{0}$ & 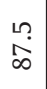 & & $\begin{array}{l}\text { 어 } \\
\text { ம் }\end{array}$ \\
\hline 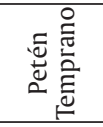 & 앗 & 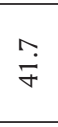 & $\begin{array}{l}0 \\
\infty \\
\infty\end{array}$ & $\stackrel{0}{0}$ & $\stackrel{m}{m}$ & $\stackrel{+}{\vec{N}}$ & ஸै & $\stackrel{+}{\grave{v}}$ & ஜ̊ & 웃 & : & : & $\begin{array}{l}\text { வें } \\
\text { }\end{array}$ & 岗 & $\stackrel{m}{\infty}$ & & $\stackrel{\circ}{\dot{m}}$ \\
\hline 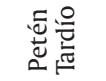 & $\bar{\sigma}$ & 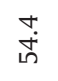 & 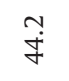 & $\stackrel{\sim}{\tilde{F}}$ & $\stackrel{\infty}{+}$ & $\stackrel{\circ}{\grave{N}}$ & $\stackrel{\infty}{\stackrel{0}{0}}$ & $\stackrel{9}{\infty}$ & 금 & $\stackrel{\llcorner}{N}$ & $\begin{array}{l}\text { กุ } \\
\text { ติ่ }\end{array}$ & $\begin{array}{l}0 \\
\tilde{\sigma}\end{array}$ & $\frac{0}{\operatorname{m}}$ & $\begin{array}{l}\stackrel{0}{ } \\
\stackrel{\text { N}}{ }\end{array}$ & $\overrightarrow{\dot{H}}$ & & $\begin{array}{l}0 \\
8 \\
0\end{array}$ \\
\hline$\breve{\Xi}$ & $\stackrel{\circ}{\dot{8}}$ & $\stackrel{0}{8}$ & $\begin{array}{l}\text { ம் } \\
\text { ம் }\end{array}$ & 吕 & $\stackrel{\circ}{\circ}$ & $\stackrel{m}{\infty}$ & 吕 & $\stackrel{\sim}{\infty}$ & O̊. & $\stackrel{+}{i}$ & $\stackrel{\circ}{\stackrel{\text { }}{ }}$ & $\stackrel{m}{m}$ & 字 & $\begin{array}{l}\circ \\
\dot{\circ}\end{array}$ & $\stackrel{\substack{n \\
\infty}}{\infty}$ & & $\ddot{8}$ \\
\hline $\begin{array}{l}\frac{\tilde{g}}{\bar{v}} \\
\frac{\bar{\sigma}}{\tilde{\sigma}}\end{array}$ & 웃 & $\stackrel{\circ}{\circ}$ & $\underset{\text { N̦}}{\text { N }}$ & $\begin{array}{l}\text { ஜें } \\
\text { }\end{array}$ & $\underset{m}{m}$ & $\bar{\sigma}$ & $\hat{g}$ & $\stackrel{+}{\grave{\Delta}}$ & $\stackrel{n}{\stackrel{n}{m}}$ & $\begin{array}{l}\infty \\
\infty \\
0 \\
0\end{array}$ & $\begin{array}{l}0 \\
\stackrel{0}{N}\end{array}$ & : & $\underset{\text { ঙิ }}{\stackrel{\mathscr{F}}{ }}$ & 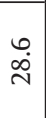 & $\underset{\infty}{\stackrel{1}{\infty}}$ & & $\stackrel{\sim}{+}$ \\
\hline $\begin{array}{l}\breve{u} \\
\infty \\
\overline{0} \\
z\end{array}$ & $\stackrel{0}{\dot{8}}$ & 亏 & $\stackrel{\stackrel{m}{+}}{+}$ & $\underset{\text { I }}{\mathcal{G}}$ & $\stackrel{\circ}{\circ}$ & $\begin{array}{l}\text { 오 } \\
\stackrel{\text { N }}{ }\end{array}$ & O̊. & 옴 & 옴 & $\vec{\pi}$ & $\stackrel{n ?}{\stackrel{n}{m}}$ & 옴 & $\stackrel{\circ}{0}$ & $\stackrel{+}{\dot{J}}$ & 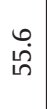 & & $\stackrel{\mathscr{\vartheta}}{\underset{f}{7}}$ \\
\hline $\begin{array}{l}\stackrel{\mathscr{U}}{\Xi} \\
\overline{0} \\
\text { z }\end{array}$ & $\stackrel{\Upsilon}{\wedge}$ & $\begin{array}{l}\stackrel{n}{\text { ஸे }} \\
\text { }\end{array}$ & 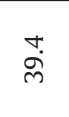 & $\stackrel{\text { ำ }}{\sim}$ & $\overline{\text { in }}$ & $\stackrel{+}{\stackrel{\sim}{N}}$ & $\begin{array}{l}\infty \\
\infty \\
\infty \\
i\end{array}$ & 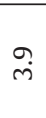 & $\stackrel{\circ}{0}$ & ํㅗㅁ & $\stackrel{0}{\circ}$ & $\stackrel{\circ}{\circ}$ & $\stackrel{\varphi}{\varphi}$ & 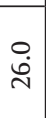 & $\begin{array}{l}\infty \\
\dot{\phi} \\
\infty\end{array}$ & & $\stackrel{0}{0}$ \\
\hline 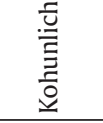 & ஜ் & ğ & $\hat{\emptyset}$ & ஜ̊ & $\bar{\sigma}$ & $\begin{array}{l}0 \\
\dot{q}\end{array}$ & 官 & $\stackrel{+}{+}$ & $\hat{\theta}$ & 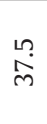 & 롬 & $\stackrel{\mathscr{L}}{ٍ}$ & $\stackrel{0}{\circ}$ & $\underset{\mathcal{H}}{\stackrel{f}{ }}$ & $\hat{\infty}$ & & $\stackrel{0}{\dot{8}}$ \\
\hline 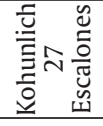 & $\stackrel{0}{\dot{8}}$ & $\frac{N}{\mp}$ & 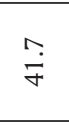 & $\begin{array}{c}m \\
\infty \\
\infty\end{array}$ & $\stackrel{\stackrel{n}{I}}{I}$ & $\begin{array}{l}\infty \\
\stackrel{\infty}{n}\end{array}$ & $\tilde{3}$ & $\hat{\sigma}$ & 菂 & 웃 & $\stackrel{\Im}{\bar{\gamma}}$ & $\stackrel{\circ}{\circ}$ & $\stackrel{0}{0}$ & 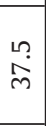 & $\stackrel{0}{\circ}$ & & $\begin{array}{l}\text { ㅇ. } \\
\text { เू. }\end{array}$ \\
\hline . & ॄ゙ & $\stackrel{\infty}{n ்}$ & $\stackrel{0}{0}$ & 今̊ & $\hat{m}$ & $\stackrel{\dddot{m}}{\sim}$ & ๓̊ & $\stackrel{\text { กุ }}{\text { กิ }}$ & $\stackrel{5}{\bar{n}}$ & $\hat{j}$ & $\hat{\theta}$ & 官 & $\stackrel{\circ}{\stackrel{\text { }}{ }}$ & $\frac{\sim}{m}$ & $\stackrel{n}{m}$ & & 華 \\
\hline 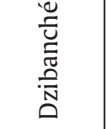 & デ & $\begin{array}{c}\text { aे } \\
\infty \\
\infty\end{array}$ & $\stackrel{n}{\stackrel{n}{m}}$ & ชิ & $\overrightarrow{\bar{N}}$ & $\begin{array}{l}\infty \\
\stackrel{\rho}{0} \\
\text {. }\end{array}$ & فํ & $\hat{\sigma}$ & $\stackrel{+}{\dot{\sim}}$ & $\stackrel{m}{\tilde{n}}$ & : & $\frac{\dot{\nabla}}{\grave{N}}$ & $\hat{\sigma}$ & $\stackrel{\mathscr{m}}{ \pm}$ & $\stackrel{m}{\tilde{n}}$ & & $\ddot{8}$ \\
\hline$\frac{\bar{\Xi}}{\frac{\bar{E}}{\sqrt{J}}}$ & $\stackrel{\circ}{\stackrel{+}{\infty}}$ & 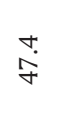 & $\stackrel{m}{\tilde{m}}$ & $\stackrel{\infty}{\stackrel{\infty}{N}}$ & $\begin{array}{l}\text { ָे } \\
\text { ָे }\end{array}$ & 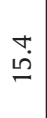 & 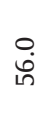 & : & $\stackrel{+}{\stackrel{+}{+}}$ & 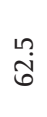 & $\stackrel{+}{\stackrel{t}{R}}$ & $\stackrel{m}{\tilde{n}}$ & $\stackrel{\sim}{\infty}$ & $\stackrel{\circ}{\stackrel{\sim}{N}}$ & 움 & & $\stackrel{m}{m}$ \\
\hline 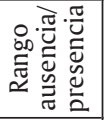 & 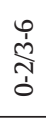 & 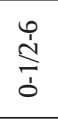 & $\delta$ & 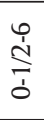 & $\delta$ & $\stackrel{?}{\frac{1}{1}}$ & $\frac{\grave{d}}{\grave{d}}$ & 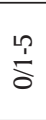 & 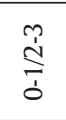 & 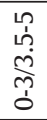 & $\underset{\substack{S \\
亡}}{\stackrel{n}{n}}$ & 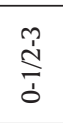 & $\frac{\stackrel{m}{S}}{0}$ & 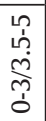 & 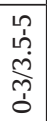 & & $\frac{\mathfrak{n}}{\stackrel{d}{c}}$ \\
\hline 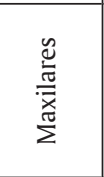 & $\begin{array}{l}\frac{\pi}{\pi} \\
\ddot{\Xi} \\
\ddot{\Xi}\end{array}$ & 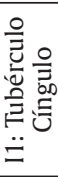 & 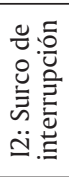 & 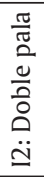 & 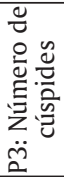 & 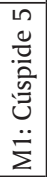 & 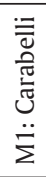 & 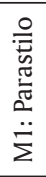 & 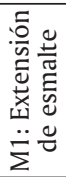 & 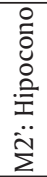 & 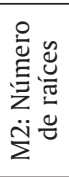 & 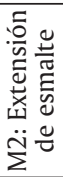 & 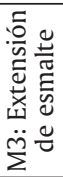 & 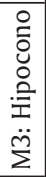 & 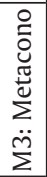 & 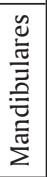 & $\begin{array}{l}\frac{\pi}{\sqrt{\pi}} \\
\ddot{\Xi} \\
\ddot{\underline{U}}\end{array}$ \\
\hline
\end{tabular}




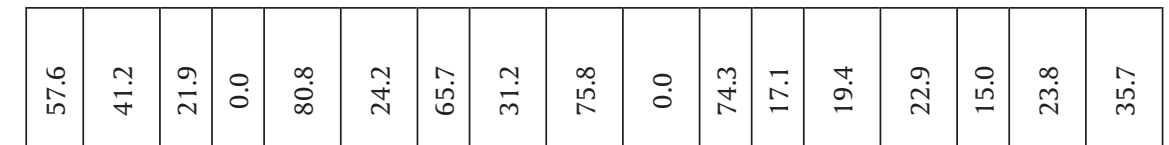

\begin{tabular}{|c|c|c|c|c|c|c|c|c|c|c|c|c|c|c|c|c|}
\hline 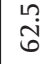 & $\underset{\tilde{N}}{\tilde{N}}$ & $\hat{\sigma}$ & $\stackrel{\infty}{+}$ & 足 & $\begin{array}{l}N \\
\infty\end{array}$ & $\underset{\infty}{\stackrel{1}{\infty}}$ & $\hat{\emptyset}$ & $\begin{array}{l}+ \\
\infty \\
0\end{array}$ & $\stackrel{\uparrow}{\wedge}$ & 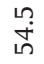 & $\bar{\sigma}$ & సુ & $\stackrel{\vec{m}}{\dot{m}}$ & $\hat{\sigma}$ & $\hat{\sigma}$ & $\stackrel{m}{m}$ \\
\hline
\end{tabular}

\begin{tabular}{|c|c|c|c|c|c|c|c|c|c|c|c|c|c|c|c|c|}
\hline $\bar{i}$ & 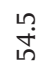 & 字 & $\because$ & $\vec{\gamma}$ & $\stackrel{\mathscr{m}}{ \pm}$ & نூ & $\underset{\dot{f}}{\vec{f}}$ & $\begin{array}{l}+ \\
0 \\
0\end{array}$ & $\overbrace{\infty}^{\infty}$ & $\stackrel{\operatorname{Ln}}{\mathrm{S}}$ & $\stackrel{\stackrel{n}{I}}{\stackrel{N}{N}}$ & $\hat{\sigma}$ & $\underset{m}{\stackrel{n}{m}}$ & $\begin{array}{l}0 \\
\text { வ் }\end{array}$ & $\stackrel{\hat{\sigma}}{\sigma}$ & 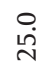 \\
\hline
\end{tabular}

\begin{tabular}{|c|c|c|c|c|c|c|c|c|c|c|c|c|c|c|c|c|}
\hline ڤே. & 움 & $\stackrel{m}{m}$ & $\stackrel{+}{\stackrel{H}{*}}$ & ஜ் & $\stackrel{\text { ஸ̣ }}{\circ}$ & $\stackrel{\text { ని }}{0}$ & $\stackrel{m}{m}$ & 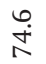 & $\tilde{n}$ & $\frac{9}{n}$ & $\underset{\infty}{\sigma}$ & $\overline{6}$ & $\hat{\text { fi }}$ & 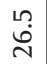 & ஸै & $\stackrel{\mathscr{n}}{\mathrm{n}}$ \\
\hline
\end{tabular}

\begin{tabular}{|c|c|c|c|c|c|c|c|c|c|c|c|c|c|c|c|c|}
\hline$\underset{\mathscr{f}}{\mathscr{f}}$ & $\stackrel{\mathscr{I}}{\mathcal{H}}$ & 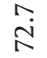 & $\stackrel{\circ}{\circ}$ & $\begin{array}{l}\text { Oें } \\
\text { in }\end{array}$ & @े & $\begin{array}{c}\text { m. } \\
\tilde{\infty}\end{array}$ & $\stackrel{ }{\mathrm{N}}$ & $\hat{g}$ & $\stackrel{0}{0}$ & $\underset{\infty}{\stackrel{1}{\infty}}$ & $\stackrel{\circ}{\circ}$ & $\stackrel{\circ}{0}$ & $\stackrel{10}{I}$ & $\stackrel{\widetilde{N}}{\text { N }}$ & $\begin{array}{l}0 \\
\text { L } \\
\text { مी }\end{array}$ & $\stackrel{m}{m}$ \\
\hline
\end{tabular}

\begin{tabular}{|c|c|c|c|c|c|c|c|c|c|c|c|c|c|c|c|c|}
\hline $\begin{array}{l}0 \\
\text { Lे } \\
\text { Ln }\end{array}$ & 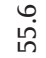 & $\stackrel{0}{\dot{q}}$ & $\stackrel{\circ}{0}$ & $\begin{array}{l}\stackrel{1}{0} \\
\infty \\
m\end{array}$ & $\stackrel{\text { In }}{\stackrel{0}{0}}$ & $\underset{\infty}{\stackrel{\Delta}{i}}$ & 임 & $\bar{\sigma}$ & $\stackrel{0}{\circ}$ & $\hat{q}$ & $\stackrel{\circ}{0}$ & $\stackrel{\circ}{\circ}$ & $\stackrel{+}{\forall}$ & $\stackrel{\circ}{\stackrel{N}{N}}$ & $\begin{array}{l}0 \\
\stackrel{\infty}{\sim}\end{array}$ & $\stackrel{\circ}{\stackrel{\sim}{े}}$ \\
\hline
\end{tabular}

\begin{tabular}{|c|c|c|c|c|c|c|c|c|c|c|c|c|c|c|c|c|}
\hline $\begin{array}{l}0 \\
100 \\
12\end{array}$ & 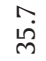 & $\hat{\sigma}$ & $\stackrel{\circ}{\circ}$ & $\stackrel{\infty}{\stackrel{\infty}{N}}$ & $\bar{\sigma}$ & ন & 임 & $\begin{array}{l}0 \\
\dot{\sigma}\end{array}$ & $\stackrel{\mathscr{n}}{\stackrel{N}{N}}$ & O̊ & @̊ं. & $\stackrel{\sim}{\infty}$ & $\stackrel{m}{m}$ & $\begin{array}{l}\stackrel{0}{0} \\
\dot{m}\end{array}$ & $\stackrel{\sim}{\infty}$ & $\begin{array}{l}0 \\
\stackrel{\sim}{N}\end{array}$ \\
\hline
\end{tabular}

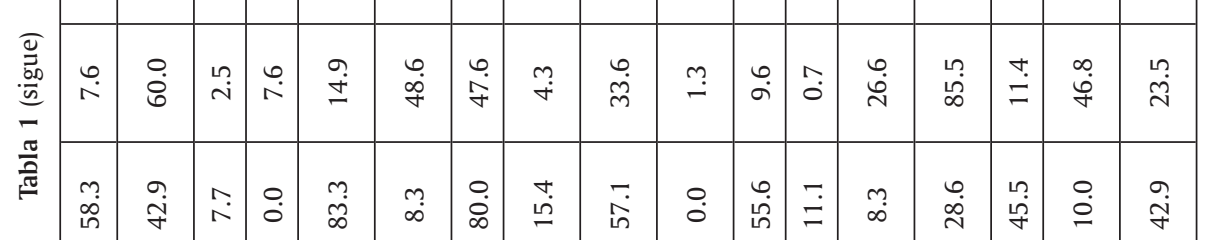

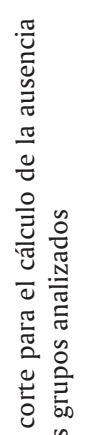
응 先 중 垔离 을 뚠

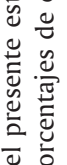

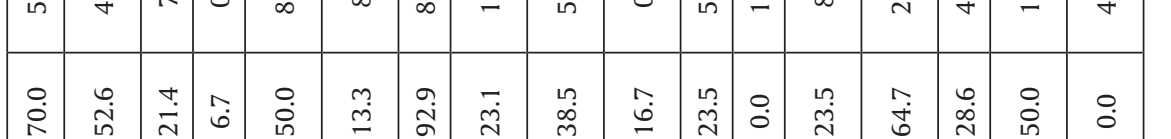

\begin{tabular}{|c|c|c|c|c|c|c|c|c|c|c|c|c|c|c|c|c|}
\hline $\begin{array}{l}0 \\
\text { O̊ }\end{array}$ & $\stackrel{m}{m}$ & $\begin{array}{l}\dot{b} \\
\dot{L}\end{array}$ & $\stackrel{+}{-}$ & $\begin{array}{l}\stackrel{0}{f} \\
\stackrel{f}{*}\end{array}$ & $\stackrel{\text { n̊ }}{\stackrel{N}{ }}$ & 号 & $\begin{array}{l}\infty \\
\infty \\
\infty\end{array}$ & $\begin{array}{l}+ \\
\text { in }\end{array}$ & $\bar{r}$ & กั & ஸु & $\infty$ & $\stackrel{\text { ñ }}{\dot{m}}$ & $\begin{array}{l}0 \\
\infty \\
\stackrel{\sim}{N}\end{array}$ & $\begin{array}{l}0 \\
\text { Ni }\end{array}$ & $\stackrel{m}{ \pm}$ \\
\hline
\end{tabular}

\begin{tabular}{|c|c|c|c|c|c|c|c|c|c|c|c|c|c|c|c|c|}
\hline 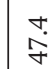 & $\frac{9}{6}$ & mे & $\stackrel{\Re}{\dot{\Xi}}$ & $\underset{\infty}{+}$ & $\hat{m}$ & $\begin{array}{l}9 \\
\infty \\
\infty\end{array}$ & ले & $\stackrel{\circ}{\circ}$ & $\stackrel{0}{0}$ & $\stackrel{n}{n}$ & 字 & $\stackrel{\infty}{+}$ & ஸे & $\begin{array}{c}m \\
\text { Ln }\end{array}$ & $\stackrel{\infty}{=}$ & 움 \\
\hline
\end{tabular}

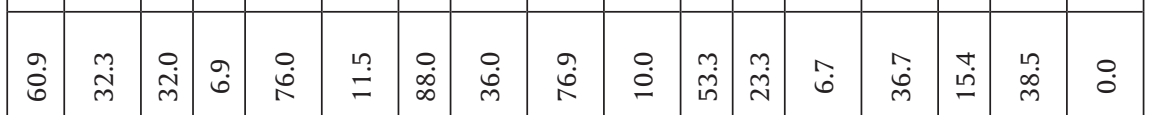

\begin{tabular}{|c|c|c|c|c|c|c|c|c|c|c|c|c|c|c|c|c|}
\hline $\begin{array}{l}\stackrel{0}{1} \\
\stackrel{1}{c}\end{array}$ & 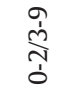 & 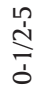 & $\frac{+}{\delta}$ & $\underset{\dot{d}}{\stackrel{2}{d}}$ & $\delta$ & $\frac{\stackrel{+}{d}}{\stackrel{d}{b}}$ & $\begin{array}{l}0 \\
b \\
*\end{array}$ & 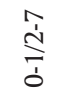 & $\curvearrowright$ & $\begin{array}{l}\text { L } \\
\text { న̂ } \\
\text { ș }\end{array}$ & 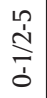 & $\begin{array}{l}\overrightarrow{+} \\
+ \\
\dot{x}\end{array}$ & $\stackrel{+}{\stackrel{+}{\wedge}}$ & 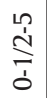 & $\stackrel{+}{\stackrel{+}{\wedge}}$ & $\underset{x}{\bar{x}}$ \\
\hline $\begin{array}{l}\stackrel{\alpha}{\Delta} \\
\ddot{\sigma}\end{array}$ & 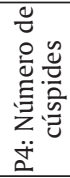 & 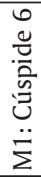 & 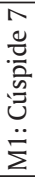 & 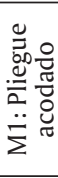 & 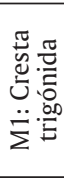 & $\begin{array}{l}\tilde{U} \\
\ddot{0} \\
\ddot{\ddot{\Sigma}}\end{array}$ & 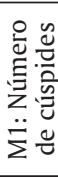 & 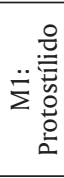 & 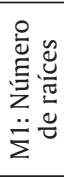 & 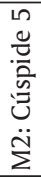 & 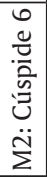 & 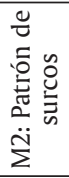 & 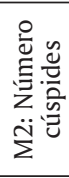 & 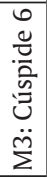 & 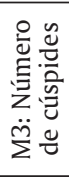 & 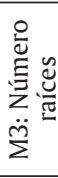 \\
\hline
\end{tabular}


dentes de las metrópolis urbanas, con un flujo elevado de personas y familias, mientras que valores reducidos atestiguan poblaciones más endógamas y aisladas. Para evaluar las afinidades y distancias biológicas entre las poblaciones, procesamos las frecuencias dicotómicas mediante una serie de análisis de variables múltiples, concretamente análisis de conglomerado (método de Ward y UPGMA) y de la Máxima Semejanza. La decisión de emplear una muestra externa de control (Neo) se basa en la necesidad de considerar en la Máxima Semejanza una muestra externa como término de comparación (Phylip 3.5; Felsenstein, 1973). Debido a que cada metodología estadística se basa en un algoritmo que mide distancias diferentes de los demás, la aplicación de más de una medición estadística permite valorar y apreciar los patrones que se repiten en cada una, y que por esta razón reflejan semejanzas reales o diferencias, que de otras formas podrían ser simplemente un artefacto estadístico. En este sentido, debido a que la Máxima Semejanza requiere de un outlier (o muestra independiente, de control) se decidió utilizarlo también en los dos análisis de conglomerado (Ward y UPGMA).

\section{La diversidad poblacional interna}

La variabilidad interna de cada grupo se calculó a partir de las frecuencias de todos los 79 rasgos analizados (tabla 2). Cabe mencionar que para esta medición se ha empleado el total de los 79 rasgos en lugar de limitarse a los que cada vez se escogen para los análisis estadísticos (en este caso, 33). Esto se debe a la necesidad de obtener un dato objetivo, único, constante y replicable en cada colección que sea comparable con los valores obtenidos en las demás series esqueléticas.

Como podemos apreciar en la tabla, a la población esquelética de Calakmul le corresponde el valor más alto que va a la par con la de Toniná, otro centro urbano que además mantenía lazos con diversas poblaciones en la zona limítrofe del territorio maya. Sigue en diversidad la ciudad de Palenque, o más bien la población de su centro urbano que fue objeto de este estudio, y luego Noh Bec (un sitio de transición), Dzibanché y finalmente Kohunlich. Sorprende a primera vista que los sitios costeros manifiesten una diversidad biológica similar a la de los grandes centros, pero se entiende por la elevada movilidad de las poblaciones costeras: por ello los separamos de la primera lista. ${ }^{4}$

Ya hemos comentado que el índice de heterocigosidad expresa la variabilidad interna de una población, la cual se da en función del intercambio entre diferentes poblaciones. Los valores obtenidos en poblaciones humanas del pasado van de 0.10 (muy homogéneo) a 0.40 (extremadamente variable). Como hemos mostrado en otro estudio, las poblaciones mayas muestran un aumento en la variabilidad interna durante las épocas de transición (como es el Clásico Terminal)

\footnotetext{
${ }^{4}$ En el caso de Xcambó, un pequeño puerto localizado en el litoral de la costa norte de Yucatán, la proporción foránea de la población funeraria (es decir, la que nació en otra región) alcanza un dramático quince por ciento (Price, comunicación personal, 2008).
} 


\begin{tabular}{|l|l|}
\hline \multicolumn{2}{|c|}{ Sitios tierra adentro } \\
\hline Petén Temprano & 0.309 \\
\hline Calakmul & 0.267 \\
\hline Toniná & 0.267 \\
\hline Petén Tardío & 0.264 \\
\hline Puuc & 0.257 \\
\hline Palenque & 0.255 \\
\hline Noh Bec & 0.249 \\
\hline Dzibanché & 0.245 \\
\hline Kohunlich & 0.220 \\
\hline Kohunlich 27 Escalones & 0.203 \\
\hline \multicolumn{2}{|c|}{ Sitios costeros } \\
\hline Xcambó & 0.264 \\
\hline Jaina & 0.252 \\
\hline
\end{tabular}

Tabla 2. Valores de variabilidad interna de los grupos analizados

(Cucina y Tiesler, 2008). Las dinámicas de movilidad y migración parecen enriquecer las poblaciones autóctonas introduciendo nuevas frecuencias y aumentando el nivel de variabilidad interna del grupo.

Más homogéneas se presentan las poblaciones relativamente estables, como muchas del Clásico, un aspecto relacionado con los fenómenos genéticos que acompañan los efectos de "cuello de botella" o "deriva génica" de una población. Para los fines de este trabajo basta decir que las grandes ciudades del Clásico generalmente se ubican por encima de 0.254 , indicando una y otra vez que sus poblaciones eran dinámicas y esencialmente exógamas. Para ofrecer una comparación, la población multiétnica de Campeche marca un índice de 0.33 . Por otra parte, si agrupamos las poblaciones esqueléticas italianas del neolítico alcanzan un índice que rodea el 0.2 , equivalente a las poblaciones mayas aldeanas tierra dentro en tiempos de estabilidad.

\section{Las relaciones fenotípicas interpoblacionales}

Igualmente hemos recurrido a las poblaciones del neolítico europeo como muestras de control (outliers) en la evaluación de distancias o afinidades fenotípicas 
entre poblaciones, la cual concede una mirada que complementa la de su variabilidad biológica interna. Como mencionamos anteriormente, los rasgos morfológicos dentales han sido elaborados estadísticamente por medio de los Análisis de Conglomerados y de la Máxima Semejanza. Para el caso del Análisis de Conglomerados, la figura 4 expresa las congregaciones obtenidas por el método de agrupación de Ward. Podemos apreciar que la muestra externa de control del neolítico europeo cumple con su función y genera dos subgrupos mayances. Una contiene a Palenque y a los dos grupos de Kohunlich, mientras que la otra agrupa las muestras restantes. En este último interesa notar que Calakmul se vincula con los sitios del Petén al sur, y con las poblaciones del norte de Yucatán de Xcambó y Jaina. Ligeramente más alejados se encuentran Dzibanché (que no se asocia a Kohunlich a pesar de la cercanía geográfica), el Puuc y Toniná. Una estadística alterna es el aglomerado por UPGMA (Unweighted Pair-Group Mean Average; figura 5). En este caso, al grupo de Palenque y Kohunlich se asocia también el Puuc, mientras que entre los demás Calakmul confirma su cercanía con Xcambó y con los del Petén, y Dzibanché su lejanía de Kohunlich.

Los resultados de la estadística de Máxima Semejanza se representan en forma de un árbol sin raíz (Felsenstein, 1973) en el cual pueden apreciarse algunas tendencias (figura 6). En primer lugar el grupo outlier del neolítico se separa por medio de una rama muy larga, al indicar la distancia existente entre él y los

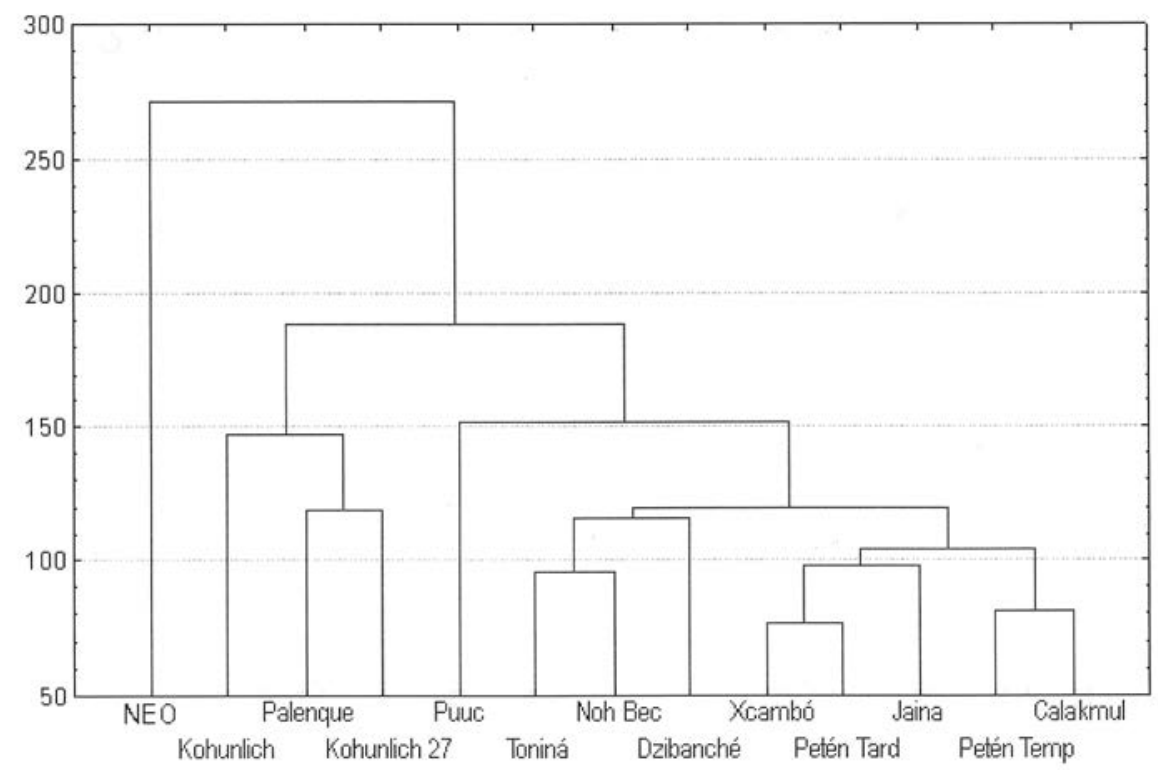

Figura 4. Resultados del análisis de conglomerados en poblaciones mayas del periodo Clásico (Método de Ward; véanse las ubicaciones en el mapa de la figura 1) 


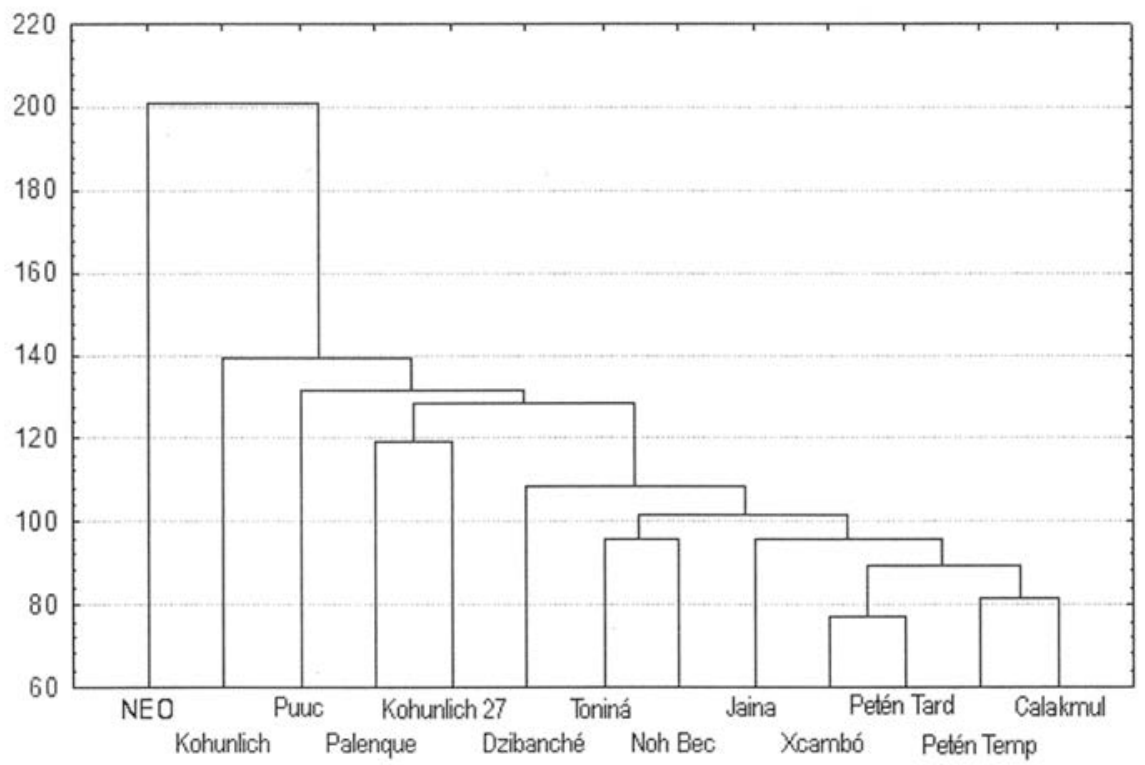

Figura 5. Resultados del análisis de conglomerados en poblaciones mayas del periodo Clásico (Método de UPGMA; véanse las ubicaciones en el mapa de la figura 1)

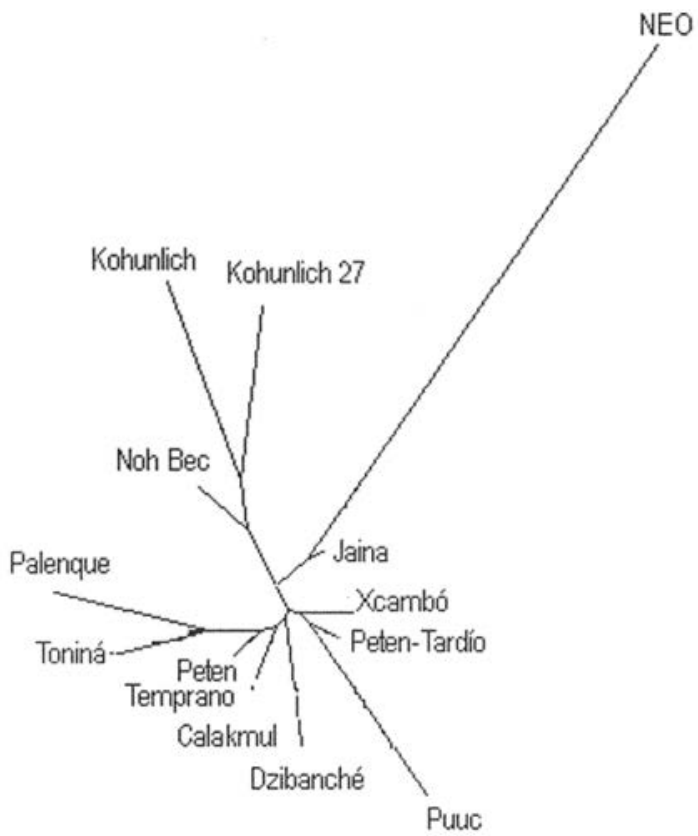

0.1

Figura 6. Análisis de Máxima Semejanza comparando poblaciones mayas del periodo clásico 
demás. Nuevamente, ambos periodos del Petén guatemalteco se acercan a Calakmul junto con Xcambó, Dzibanché y el Puuc, mientras que los dos grupos de Kohunlich se alejan de los demás. Aunque comparten la misma rama, Palenque y Toniná se separan de los sitios del Petén y del norte de Yucatán, indicando una cercanía morfológica menos patente respecto a los demás.

En general, los resultados morfológicos dentales evidencian una homogeneidad biológica que refleja los resultados obtenidos por Austin (1978) y Pompa (1984) para este mismo periodo, aunque hayan sido obtenidos con métodos diferentes. Según pensamos, esta uniformidad es el producto de una continuidad poblacional entre los grupos que residían en Yucatán y el Petén durante el periodo Clásico. Aunque estos datos solo trazan tendencias, los resultados atestiguan una elevada dinámica poblacional al interior del amplio corredor que atravesaba las Tierras Bajas, como lo subraya también Sharer (1994) a partir de otras fuentes de información. La movilidad de los grupos no debe haberse limitado al entorno de las comunidades, regiones o unidades políticas; aquellos se distribuirían dinámicamente a lo largo del territorio. A conclusiones similares llega Rodrigo Liendo (2008) a partir de una evaluación de poblaciones y asentamientos en la región de Palenque. Andrew Scherer (2004; 2007), por su lado, excluye — tras un análisis de rasgos dentales métricos y morfológicos en la Tierras Bajas del Clásico- que el aislamiento por distancia represente un factor micro-evolutivo que haya caracterizado las antiguas poblaciones mayas de las Tierras Bajas. Esta visión refleja también las evidencias epigráficas sobre territorialidad y etnicidad durante el Clásico (Lacadena y Wichman, 2002; Martin y Grube, 2008: 17-21); y encuentra su confirmación en los estudios isotópicos de estroncio. Los recientes resultados, obtenidos en Copán, Tikal, Palenque y el mismo Calakmul (Price et al., 2006; 2008; White, Price y Longstaffe, 2007), apuntan a que los movimientos entre áreas estaban a la orden del día no sólo entre los miembros de las dinastías peteneras sino también entre los sectores populares. Resulta interesante en este contexto notar la relación de Calakmul no sólo con sitios cercanos y grandes como Dzibanché, sino también con Noh Bec y el grupo del Puuc, ubicados más al norte y hacia el centro de la península de Yucatán. Ellos a su vez se acercan a los asentamientos costeros como Xcambó, lo cual testimonia una distribución poblacional dinámica y continua que era marcada por las redes comerciales tanto costeras como terrestres. Esta dinámica parece ser un preludio de las migraciones masivas que se dieron desde las áreas peteneras hacia el norte peninsular en las vísperas del Posclásico, las cuales conllevarían una reorganización social y biológica en esta región (Andrews et al., 2003).

En el caso de la serie calakmulense, el acercamiento reiterativo con los grupos del Petén guatemalteco del Clásico Temprano y (aunque en menor grado) del Tardío atestigua una dinámica poblacional en un corredor relativamente abierto también hacia las Tierras Altas al sur. Más en específico, el hecho de que la serie calakmulense, que data mayormente del Clásico Tardío, se acerque sobre todo a la población temprana del sureste de Petén más que a la tardía y a las series de 
Dzibanché ubicadas al este, podría ser expresión de movimientos graduales de población petenera hacia los territorios del norte, como lo sugieren Laporte y Mejía (2005: 147) al hablar de flujos de sobrepoblación hacia grandes centros foráneos.

Asimismo, resalta su patente separación con las dos agrupaciones de Kohunlich que, a su vez, se acercan a Palenque. La lejanía geográfica de este último, localizado en el territorio maya periférico al oeste del río Usumacinta, puede ser un elemento más que explique su distancia fenotípica respecto a los grupos peteneros, al tiempo que la cercanía geográfica y política con Toniná podría explicar por qué las dos series arqueológicas se agrupan. Mientras tanto, resulta difícil interpretar las condiciones poblacionales tras el distanciamiento fenotípico de Kohunlich de las series del Petén y su cercanía con las chiapanecas.

Interesa notar que en un estudio anterior (Cucina y Tiesler, 2008) notamos una lejanía del área poblacional petenera (Calakmul y el Petén guatemalteco) respecto a los grupos ubicados al este (en lo que es actualmente Belice), identificando un corredor en dirección sur-norte más que con extensión hacia el este. Desgraciadamente, los datos disponibles de Belice derivan de los de Wrobel (2004), por lo que no es posible determinar hasta dónde estos resultados puedan ser el producto del error entre observadores o reflejen distancias poblacionales reales. Así, quedará por corroborar esta idea en futuros estudios propios.

\section{La distribución local y regional de las formas cefálicas}

Las mismas series evaluadas en cuanto a su morfología dental también fueron objeto de un estudio de formas cefálicas artificiales. Nuestros resultados nos informan que la deformación cultural está presente en un promedio de 86 por ciento de los cráneos evaluables $(\mathrm{N}=722)$. Ninguno de los sitios a lo largo del corredor norte-sur peninsular muestra frecuencias de presencia por debajo de 75 por ciento (figura 7), lo cual subraya la elevada importancia que esta práctica cobraba en la vida diaria de las familias en todo el territorio bajo estudio.

Aparte del grado de popularidad de la costumbre nos interesamos por las preferencias técnicas y sus resultados finales en la morfología cefálica. Sabemos que las formas cefálicas artificialmente logradas eran muy variadas en el territorio maya durante el periodo Clásico (Stewart, 1975: 199-201; Tiesler, 1998) y pensamos que jugaban un papel emblemático entre sus portadores, fungiendo como señal visible de identidad y pertenencia étnica (posiblemente estos fueron motivados por el afán de emular la morfología de poderes naturales). Este último aspecto cobra especial importancia al reconocer las formas cefálicas populares en los retratos del panteón de los dioses, coyuntura que ya ha sido planteada por varios autores a partir de la iconografía del Clásico (Romano, 1987; Taube, 1992: 46-50, 64-69; Sotelo y Valverde, 1992; Houston, Stuart y Taube, 2006: 45).

Para los fines de este trabajo interesa saber que las poblaciones funerarias peteneras estudiadas presentan la modalidad tabular oblicua (tono gris claro en la 


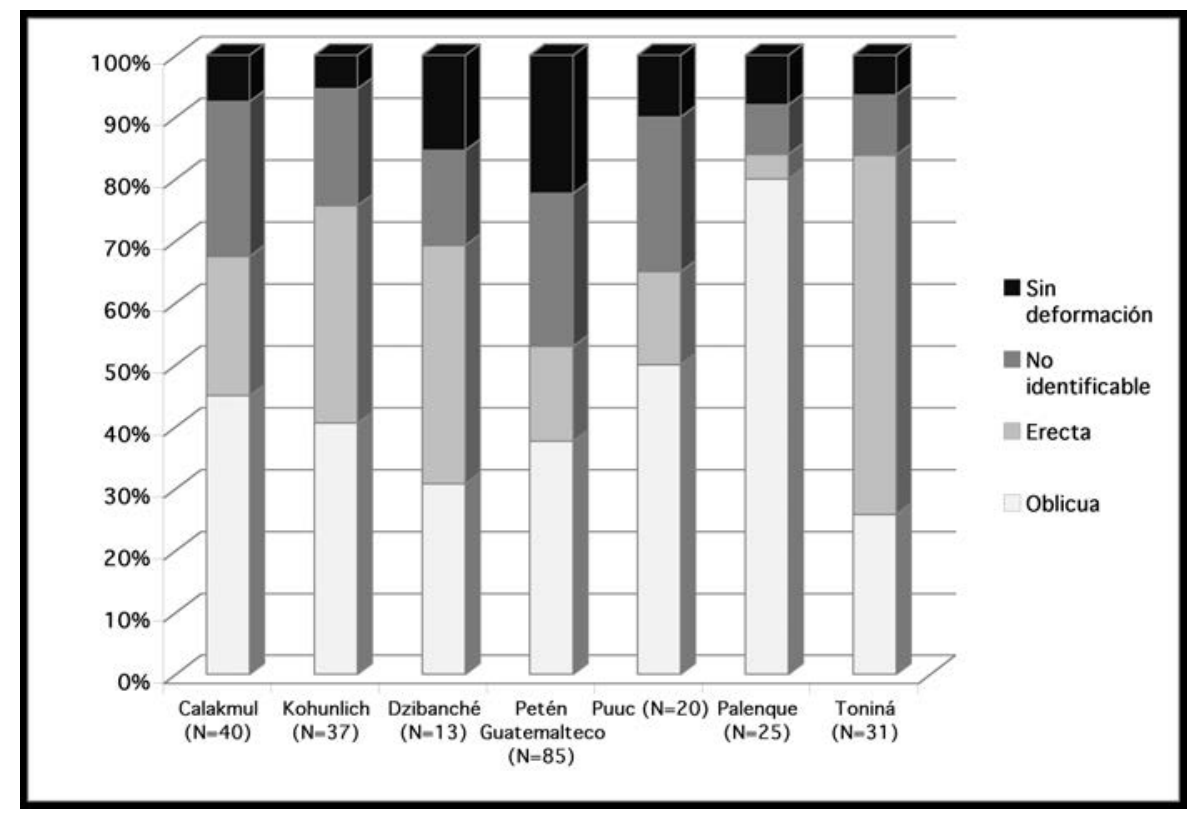

FiguRa 7. Mapa del área maya con distribución de preferencias de formas cefálicas

figuras 7) en una proporción que va del 30 al 60 por ciento respecto a la tabular erecta (tono gris medio en la figuras 7; véase también la figura 8, retomada de Tiesler, 2011). Esta relación se mantiene constante a lo largo del Clásico en los sitios de tierra adentro que permitían una comparación entre el Clásico Tardío y el Temprano. En especial el Petén Central y el Petén Norte estilan proporciones similares en todas las colecciones que pudimos analizar, manifestando una continuidad y homogeneidad cultural en los términos planteados para esta tradición. Estos hechos son consistentes en principio con la continuidad fenotípica establecida con los datos de morfología dental.

La distribución petenera de las formas cefálicas artificiales difiere notablemente de Palenque, al oeste, que estila casi exclusivamente la deformación tabular oblicua (aunque en diferentes variantes y grados), materializando las cabezas reclinadas y tubulares que conocemos de la iconografía palencana. Interesa saber que esta preferencia por aparatos cefálicos y cabezas reclinadas se extiende hacia el sur hasta el área del río de la Pasión a lo largo de la cuenca del Usumacinta (Tiesler, 1999; véase la figura 8). Desafortunadamente no tenemos una cobertura suficiente de series esqueléticas beliceñas como para poder comparar las preferencias peteneras de formas cefálicas con aquellas de las poblaciones al este de las Montañas Mayas y hacia las costas orientales beliceñas. Por ello nos limitamos a discutir las diferencias observadas entre el Petén y los territorios al oeste. 

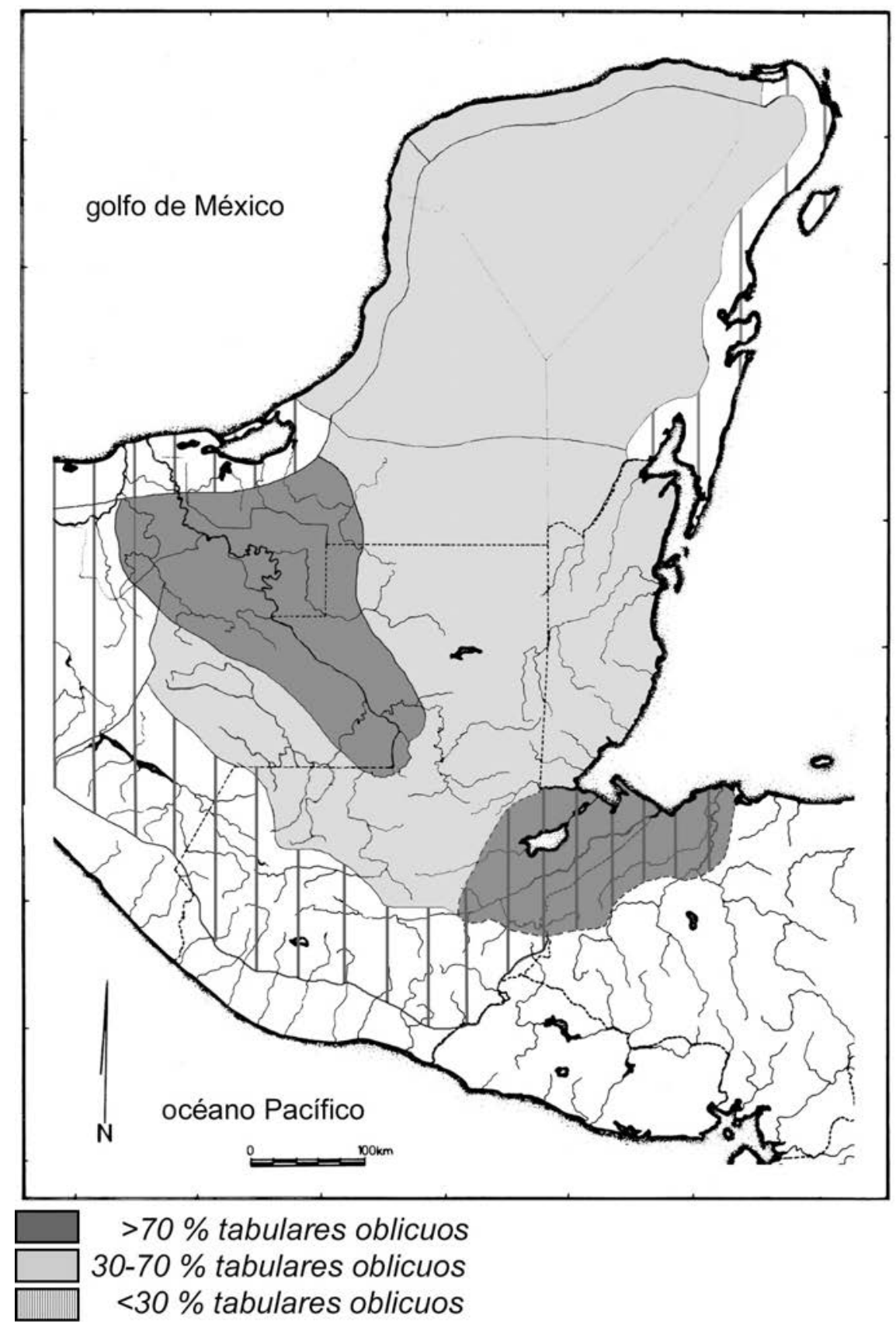

\section{$>70 \%$ tabulares oblicuos \\ 30-70\% tabulares oblicuos \\ $<30 \%$ tabulares oblicuos}

Figura 8. Preferencias de formas cefálicas en los territorios mayas durante el periodo Clásico (mapa retomado de Tiesler, 2011): la tonalidad gris oscura indica áreas con preferencias por formas cefálicas inclinadas (en comparación con erectas); el tono gris claro manifiesta el uso de ambas formas genéricas (oblicuas y erectas); las superficies estriadas delinean zonas donde se prefieren formas erectas. En el caso de Copán, se distingue la zona urbana, con fuerte preferencia por formas oblicuas, de su Hinterland rural (con preferencia por formas erectas) 
Nos preguntamos, al respecto, si las discrepancias preferenciales entre el oeste y el este/norte de las Tierras Bajas pudieran expresar una divergencia cultural más profunda, vinculada quizá con diferencias linguísticas o esquemas ideológicos distintivos (aspecto muy factible considerando lo diverso y extenso de la geografía cultural representada por las Tierras Bajas Mayas durante el periodo Clásico). En este sentido interesa notar que la distribución geográfica de los tipos cefálicos -i.e. tabulares oblicuos en la cuenca del Usumacinta y variedad de técnicas a lo largo del corredor petenero- sigue la de otras manifestaciones humanas en el área, como son las convenciones del retrato artístico (véase por ejemplo Kettunen, 2005: 182-186) o aquella de las lenguas vernáculas. Recientemente, Alfonso Lacadena y Søren Wichman (2002) han reconstruido una línea linguíística divisora de norte a sur (entre territorios de habla Ch'olan occidental y oriental), que los autores ubican en algún lugar al oeste de la región de Petexbatún, borrándose las diferencias en el habla en el área de la cuenca del río de la Pasión al sur (véase también Mora-Marín, Hopkins y Josserand, 2009).

Regresando a los residentes del corredor petenero y peninsular, donde también se emplaza el Reino de Kaan, cada uno de los sitios estudiados ostenta aquí una diversidad de formas y variantes del modelaje cefálico. Esta diversidad se observa en las series de los grandes centros como Calakmul (figura 9) y en los
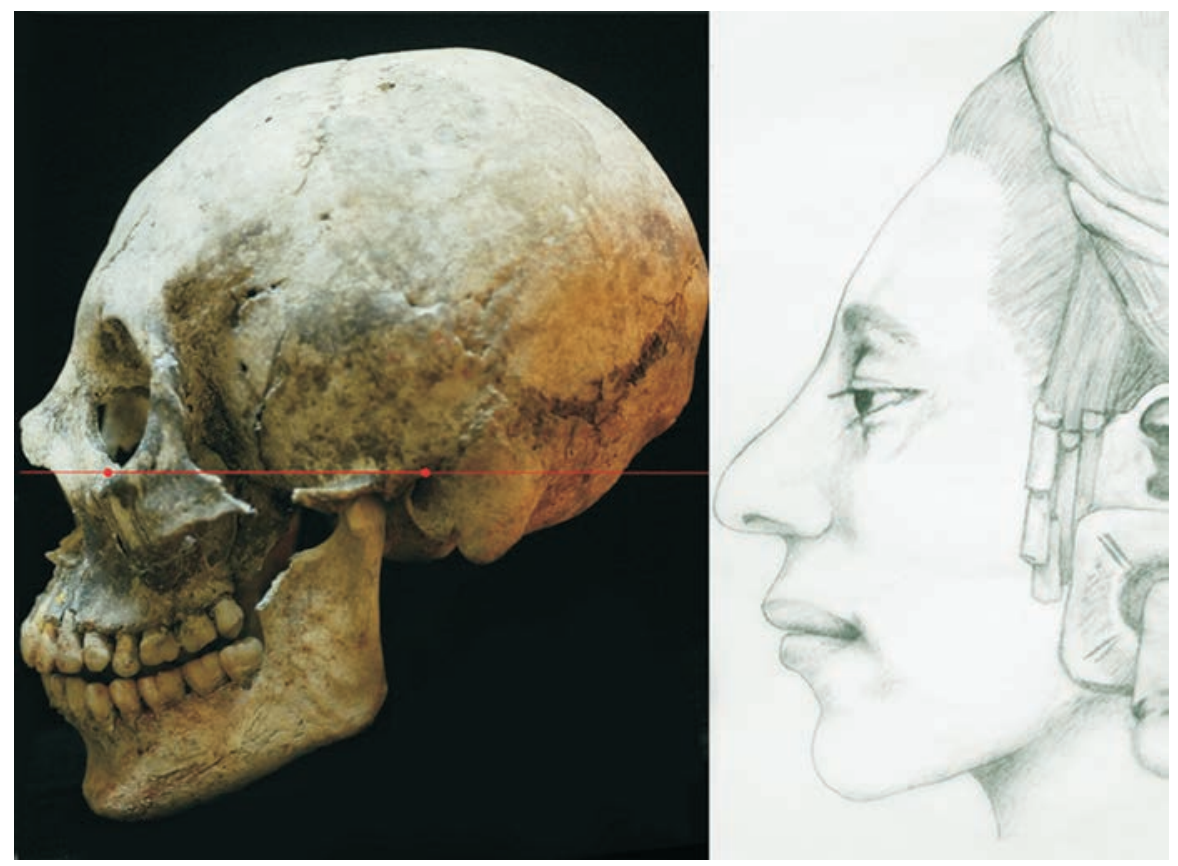

Figura 9. Norma lateral izquierda del cráneo de la acompañante del personaje sepultado en el Tumba II-4, Calakmul, Campeche (Proyecto INAH): (A) norma lateral izquierda del cráneo; (B) reconstrucción facial hipotética en perfil (foto y reconstrucción de Vera Tiesler) 
pequeños asentamientos, como los que integran la serie esquelética del Petén guatemalteco al sur (Tiesler, 2005).

Agregamos que estudios anteriores no pudieron evidenciar diferencias relevantes en las formas cefálicas femeninas y masculinas o al comparar las preferencias de los distintos sectores sociales (cuya pertenencia inferimos a partir de los ajuares funerarios). La distribución interna de las formas cefálicas culturales más bien hace pensar en su papel como medio de reproducción de tradiciones familiares, quizá linajes, aparentemente con independencia del género, del estatus o de la localidad de residencia de su portador (Tiesler, 1998, 1999, 2011). El hecho de que fueran mujeres quienes estaban a cargo de imprimir la forma deseada en las cabezas de su cría permite conocer la vida religiosa colectiva desde un ángulo poco explorado hasta el momento: la perspectiva femenina, la participación de ellas en el fomento ideológico de grupo y de identidad, instrumentos para la etnogénesis y el cambio social. Desde un punto de vista regional, la tradición en sus múltiples expresiones no parece demarcar fronteras étnicas divisoras dentro del corredor petenero: subraya el peso que tenía el dinamismo y el intercambio cultural y, dentro de ambos, la importancia del parentesco y de las relaciones horizontales entre grupos.

\section{Conclusión}

Con este estudio hemos deseado aportar a la discusión sobre el Reino de Kaan y sus vecinos una visión alterna, anclada directamente en los vestigios de sus antiguos pobladores. Las evidencias directas sobre relaciones biológicas y culturales que aportan los restos esqueléticos de las Tierras Bajas Mayas vienen a complementar la investigación arqueológica convencional, los estudios estilísticos y las lecturas epigráficas de los esquemas regionales mayas del Clásico. Como muestran los resultados, los marcadores dentales y los artificios cefálicos guardan un gran potencial para rastrear e interpretar las dinámicas poblacionales y culturales que en su momento subyacían al desenvolvimiento de la antigua sociedad, de sus sectores populares y de sus grupos protagónicos.

En concreto, las tendencias que emergen paralelamente de los análisis dentales y cefálicos de este estudio muestran ciertas semejanzas entre sí al manifestar una continuidad biocultural en las áreas céntricas de las Tierras Bajas Mayas durante el periodo Clásico. Esta tendencia sugiere, por principio, que los territorios del Reino de Kaan y sus unidades políticas vecinas no convivían lado al lado como bloques hegemónicos aislados sino que sus pobladores, y con ellos sus bagajes culturales, deben haber estado en constante contacto, movimiento e intercambio, todas características de una sociedad abierta y exógama. Menos claras son las evidencias al comparar los patrones dentales y los artificios cefálicos del corredor petenero con las poblaciones de la cuenca del río Usumacinta al oeste. Aquí, los resultados dentales no parecen confirmar la diferencia entre las 
preferencias de la modificación cefálica que constatamos al comparar el centro con el occidente del Petén. Consideramos que este aspecto pudiera indicar una separación meramente cultural sin mayores trasfondos biológicos o un origen relativamente reciente de la separación cultural constatada.

De modo general, los patrones aquí documentados hacen patente el dinamismo poblacional en la región, manifestando aspectos más profundos de la organización social y la distribución cultural en el área. La distribución de las formas cefálicas peteneras sugiere uniformidad cultural dentro el corredor petenero y al mismo tiempo subraya la importancia que los lazos familiares y las relaciones horizontales entre los grupos jugaban. De manera similar, los patrones morfológicos dentales dan fe de las dinámicas poblacionales abiertas y la movilidad de sus pobladores. En lo que toca al Reino de Kaan y sus residentes, esta visión ofrece una explicación alterna sobre la naturaleza cambiante del paisaje político que Calakmul vivía a lo largo del Clásico, un mundo hegemónico fluctuante y esencialmente inestable cuyos jerarcas intentaban, con una suerte cambiante, imponerse sobre una organización social tradicional que no se adhería a territorios concretos.

\section{BIBLIOGRAFÍA}

Andrews, Anthony P., E. Wyllys Andrews y Fernando Robles Castellanos

2003 "The northern maya collapse and its aftermath", Ancient Mesoamerica, XIV (1): 151-156. Cambridge: Cambridge University Press.

Austin, Donald M.

1978 "The biological affinity of the ancient population of Altar de Sacrificios and Seibal”, Estudios de Cultura Maya, XI: 57-73. México: Universidad Nacional Autónoma de México, Instituto de Investigaciones Filológicas, Centro de Estudios Mayas.

Blom, Deborah E.

2005 "Embodying borders: Human body modification and diversity in Tiwanaku society”, Journal of Archaeological Science, XXIV (1): 1-24. Cambridge [Reino Unido]: Elsevier.

Bonavides, Enrique

1992 "Ritos de pasaje entre los mayas antiguos", Estudios de Cultura Maya, XIX: 397-425. México: Universidad Nacional Autónoma de México, Instituto de Investigaciones Filológicas, Centro de Estudios Mayas.

Civera, Magalí, y Lourdes Márquez

1996 "Perfiles paleodemográficos de algunas poblaciones prehispánicas mesoamericanas”, La antropología física en México. Estudio sobre la población antigua y contemporánea, Sergio López Alonso, Carlos Serrano y Lourdes Márquez Morfín (eds.). México: Universidad Nacional Autónoma de México, Instituto de Investigaciones Antropológicas, pp. 153- 170. 
Cucina, Andrea y Vera Tiesler

2008 "Afinidades biológicas y dinámicas poblacionales en la península de Yucatán desde el Clásico hasta el periodo colonial”, El territorio maya. V Mesa Redonda de Palenque, Rodrigo Liendo Stuardo (coord.). México: Instituto Nacional de Antropología e Historia, 97-136.

Cucina, Andrea, Vera Tiesler y Gabriel Wrobel

2005 "Afinidades biológicas y dinámicas poblacionales mayas desde el Clásico hasta el periodo colonial", Los Investigadores de la Cultura Maya, XIII (2): 559-567. Campeche: Universidad Autónoma de Campeche.

Culbert, Patrick, y Don Rice (eds.)

1990 Precolumbian Population History in the Maya Lowlands. Albuquerque [Estados Unidos]: University of New Mexico.

Dahlberg, Albert A.

1956 Materials for the establishment of standards for classification of tooth characters, attributes and techniques in morphological studies of the dentition. Chicago: University of Chicago, Zollar Laboratory of Dental Anthropology.

Dembo, Adolfo y José Imbelloni

1938 Deformaciones intencionales del cuerpo humano de carácter étnico. Buenos Aires: Biblioteca Humanior.

Felsenstein, Joe

1973 "Maximum-likelihood estimation of evolutionary trees from continuous characters", American Journal of Human Genetics, XXV: 471-492. Cambridge [Estados Unidos]: Cell Press.

Hillson, Simon

1996 Dental anthropology. Cambridge: Cambridge University Press.

Hoppa, Robert D., y James W. Vaupel (eds.)

2002 Paleodemography. Age distribution from skeletal samples. Cambridge [Reino Unido]: Cambridge University Press.

Houston, Stephen, David Stuart y Karl A. Taube

2006 The memory of bones. Body, being and experience among the Classic Maya. Austin: University of Texas Press.

Kettunen, Harri

2005 "Nasal motifs in Maya iconography”, tesis para obtener el grado de Philosophy Doctor. Helsinki: University of Helsinki.

Lacadena, Alfonso, y Søren Wichmann

2002 "The distribution of Lowland Maya languages in the Classic Period", Memoria de la Tercera Mesa Redonda de Palenque, vol. 2, Vera Tiesler, Rafael Cobos y Merle Greene (eds.). México: Instituto Nacional de Antropología e Historia, Universidad Autónoma de Yucatán, 275-319, 2 vols. 
Laporte, Juan Pedro y Héctor Mejía

2005 La organización territorial y política en el mundo maya clásico: El caso del sureste y centro-oeste de Petén, Guatemala. Guatemala: Instituto de Antropología e Historia de Guatemala.

Larsen, Clarke S. y Mark A. Kelley

1991 "Introduction", Advances in Dental Anthropology, Mark A. Kelley y Clarke S. Larsen (eds.). Nueva York: Wiley Liss, 1-5.

Liendo Stuardo, Rodrigo

2008 "Fronteras, territorio y estructura de asentamientos en la región de Palenque, Chiapas: aspectos de método y teoría”, El territorio maya. V Mesa Redonda de Palenque, Rodrigo Liendo Stuardo (coord.). México: Instituto Nacional de Antropología e Historia, 401417.

Martin, Simon y Nikolai Grube

2008 Chronicle of the Maya kings and queens, 2a ed. Nueva York: Thames and Hudson.

Martínez Muriel, Alejandro

2008 "Demografía maya”, El territorio maya. V Mesa Redonda de Palenque, Rodrigo Liendo Stuardo (coord.). México: Instituto Nacional de Antropología e Historia, 475-492.

Mora-Marín, David, Nicholas Hopkins y Kathryn Josserand

2009 "The Linguistic Affiliation of Classis Lowland Mayan Writing and the Historical Sociolinguistic Geography of the Mayan Lowlands", The Ch'orti' Maya Area. Past and Present, Brent E. Metz, Cameron L. McNeil y Kerry M. Hull (eds.). Gainesville: University Press of Florida, 15-28.

Nichol, Christian R.

1989 "Complex segregation analysis of dental morphological variants", American Journal of Physical Anthropology, LXXVIII (1): 37-59. Nueva York: Wiley Liss.

Pompa y Padilla, José Antonio

1984 "Jaina y Chichén Itzá: morfología dentaria normal de dos muestras de la población maya prehispánica”, Investigaciones Recientes en el Área Maya, XVII Mesa Redonda, San Cristóbal de las Casas: Sociedad Mexicana de Antropología, 481-489.

Price, T. Douglas, Vera Tiesler, James H. Burton y Jane Buikstra

2006 "Isótopos y tumbas reales en Copan, Palenque y Calakmul”, Los Investigadores de la Cultura Maya, XIV (1): pp. 87-93. Campeche: Universidad Autónoma de Campeche.

Price, T. Douglas, James H. Burton, P. D. Fullagar, Lori E. Wright, Jane E. Buikstra y Vera Tiesler

2008 "Strontium isotopes and human mobility in ancient Mesoamerica", Latin American Antiquity, XIX (1): 167-180. Washington D.C.: Society for American Archaeology. 
Romano Pacheco, Arturo

1965 Estudio morfológico de la deformación craneana en Tamuín, S.L.P., y en la Isla del Idolo, Veracruz. México: Instituto Nacional de Antropología e Historia (Serie de Investigaciones, 10).

1987 "Iconografía cefálica maya”, Memorias del Primer Coloquio Internacional de Mayistas; México: Universidad Nacional Autónoma de México, Centro de Estudios Mayas, 1413-1474.

Sattenspiel, Lisa y Henry Harpending

1983 "Stable populations and skeletal age", American Antiquity, 48 (3): 489-498. Washington D.C.: Society for American Archaeology.

Scherer, Andrew K.

2004 "Dental analysis of classic period population variability in the Maya area", tesis para obtener el grado de Philosophy Doctor. College Station [Estados Unidos]: Texas A\&M University.

2007 "Population structure of the Classic Maya period", American Journal of Physical Anthropology, CXXXII (3): 367-380. Nueva York: Wiley Liss.

Scott, Richard G. y Christy G. Turner

1997 The Anthropology of Modern Human Teeth. Dental Anthropology and its Variation in Recent Human Populations. Cambridge: Cambridge University Press.

Sharer, Robert J.

1994 The ancient Maya, 5 ${ }^{\mathrm{a}}$ ed. Stanford: Stanford University Press.

Sharer, Robert J. y Loa P. Traxler

2006 The ancient Maya, $6^{\text {a }}$ ed. Stanford: Stanford University Press.

Sotelo, Laura y Carmen Valverde

1992 "Los señores de Yaxchilán: un ejemplo de felinización de los gobernantes mayas", Estudios de Cultura Maya, XIX: 187-214. México: Universidad Nacional Autónoma de México, Instituto de Investigaciones Filológicas, Centro de Estudios Mayas.

Stewart, T. Dale

1975 "Human Skeletal Remains from Dzibilchaltun, Yucatan, Mexico, with a Review of Cranial Deformity Types in the Maya Region", Middle American Research Institute Publication, XXXI: 199-225. Nueva Orleans: Tulane University.

Taube, Karl A.

1992 The Major Gods of Ancient Yucatan. Washington, D.C.: Dumbarton Oaks Research Library and Collection.

Tiesler, Vera

1998 La deformación cefálica intencional entre los mayas prehispánicos: aspectos morfológicos y culturales. México: Instituto Nacional de Antropología e Historia (Colección Científica). 
Tiesler, Vera

1999 "Rasgos bioculturales entre los antiguos mayas: aspectos culturales y sociales", tesis de doctorado en Antropología. México: Universidad Nacional Autónoma de México.

2005 "Prácticas bioculturales y organización social en los sitios de Copán, Honduras, y Xcambó, Yucatán”, Estudios de Antropología Biológica, XII: 635-659; Carlos Serrano, Patricia Olga Hernández y Francisco Ortiz (eds.). México: Universidad Nacional Autónoma de México, Instituto de Investigaciones Antropológicas.

2011 Transformarse en maya. El modelado cefálico entre los mayas prehispánicos y coloniales. México: Universidad Nacional Autónoma de México/Universidad Autónoma de Yucatán.

Torres-Rouff, Christina

2002 "Cranial vault modification and ethnicity in Middle Horizon San Pedro de Atacama, North Chile", Current Anthropology, XLIII (1): 163-171. Chicago: The University of Chicago Press.

Turner, Christy G.

1969 "Microevolutionary interpretations from the dentition", American Journal of Physical Anthropology, XXX (2): 421-426. Nueva York: Wiley Liss.

Turner, Christy G., Christian R. Nichol y Richard G. Scott

1991 "Scoring procedures for key morphological traits of the permanent dentition: the Arizona State University Dental Anthropology System", Advances in Dental Anthropology, editado por Marc A. Kelley y Clark S. Larsen. Nueva York: Wiley Liss, 13-31.

White, Christine D., T. Douglas Price, y Fred J. Longstaffe

2007 "Residential histories of the human sacrifices at the Moon Pyramid: evidence from oxygen and strontium isotopes", Ancient Mesoamerica, XVIII (1): 159-172. Cambridge: Cambridge University Press.

Wrobel, Gabriel D.

2004 "Metric and non-metric dental variation among the ancient maya of northern Belize", tesis para obtener el grado de Philosophy Doctor. Indiana [Estados Unidos]: University of Indiana. 